\title{
TUFTED FOLLICULITIS: AN EASILY MISSED ENTITY
}

Sowmya Naga Dogiparthi ${ }^{1}$, M. Krishnakanth ${ }^{2}$, S. Adikrishnan ${ }^{3}$, Adithya B ${ }^{4}$, Sudha R ${ }^{5}$

\section{HOW TO CITE THIS ARTICLE:}

Sowmya Naga Dogiparthi, M. Krishnakanth, S. Adikrishnan, Adithya B, Sudha R. "Tufted Folliculitis: An Easily Missed Entity". Journal of Evolution of Medical and Dental Sciences 2014; Vol. 3, Issue 13, March 31;

Page: 3289-3294, DOI: $10.14260 /$ jemds/2014/2282

ABSTRACT: A 28-year-old man and a 25 year old man presented with multiple swellings over the scalp. Physical examination revealed multiple bundles of hair emerging from a single dilated follicular opening. Histological examination of tufted areas showed hyperkeratosis and parakeratosis and follicular plugging with perifollicular acute and chronic inflammation. Staphylococcus aureus was found to be present in the bacterial cultures from one of the patients. The patients were treated with systemic antibacterial modalities that proved effective. The presented case shows a strong pathogenetic correlation between tufted hair folliculitis and staphylococcal infection, a finding that further supports infectious nature of the disease.

KEYWORDS: Tufted Hair Folliculitis.

INTRODUCTION: Tufted folliculitis is a rare entity of the scalp that resolves with cicatricial alopecia within multiple hair tufts emerging from dilated follicular orifices ${ }^{1}$. A mild variant of folliculitis decalvans as considered by some; it is a relapsing localized inflammatory disease of the scalp. Tufted folliculitis requires early diagnosis with prompt initiation of treatment to prevent from the consequences of scarring.

Tufting of the hair is caused by clustering of the adjacent follicular units due to the retention of telogen hairs within a dilated follicular orifice ${ }^{2}$. The various pathomechanisms proposed are: 1 . recurrent infections of the follicles- esp. Staphylococcus Aureus. 2. Nevoid abnormalities and 3. Retention of telogen hairs in the tufts ${ }^{3}$. Here we present two cases of tufted folliculitis that were effectively treated with antibacterial treatment after verifying the infectious nature of the disease.

CASE REPORT-1: A 28 year old Indian man presented to our department with a 2 year history of chronic relapsing inflammatory lesions of the scalp. He complained of intermittent itching and sticky hair over the affected area along with pain over the occipital region. There was no past medical history and he was otherwise healthy. The physical examination revealed a circumscribed area of $8 \mathrm{~cm}$ in length and $5 \mathrm{~cm}$ in width of hair loss was present on the occipital region of the neck.

Further examination revealed multiple skin colored papules with dilated follicles with emergent hair in tufts along with cicatricial alopecia over the region. Upon viewing the region with a magnifying hand lens; there were multiple tufts of 5-10 hairs arising from each hair ostium. The posterior cervical lymph nodes were palpable and tender. There were no altered laboratory parameters. A punch biopsy was done from the affected region to confirm the diagnosis. 


\section{CASE REPORT}

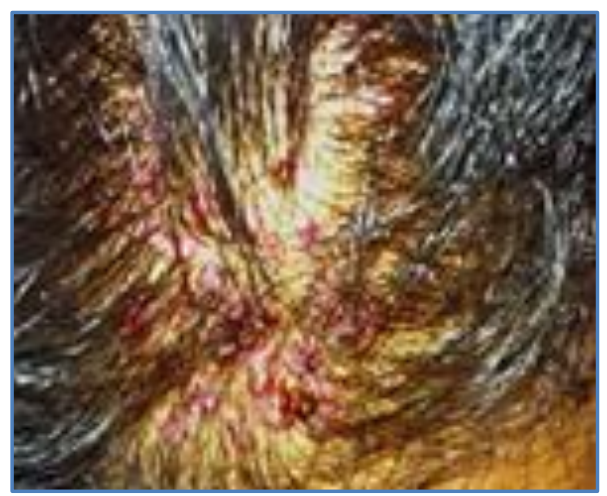

Figure 1

Scalp; Occipital region, erythematous, infiltrated plaque of scarring alopecia with dilated follicular openings with tufts containing 20 to 30 apparently normal hair shafts.

CASE REPORT- 2: Similarly, a 25 year - old Indian male presented to our out-patient department with multiple swellings over the nape of the neck associated with pain, occasional itching and discharge from the region on and off since past 3 years. Past history revealed no medical comorbidities. On general examination patient was moderately built and was of good health. Local examination revealed multiple flesh colored, dome shaped papules surmounted by 10-12 hairs emerging in tufts from the dilated follicles over the nape of the neck. The region had undergone recurrent episodes of exudation and crusting, and follicular discharge of purulent material was evident. All the laboratory parameters were within the normal limits except for the following. The pus was sent for culture and sensitivity, which revealed Staphylococcus aureus. Cultures were followed by histopathological evaluation for confirmation.

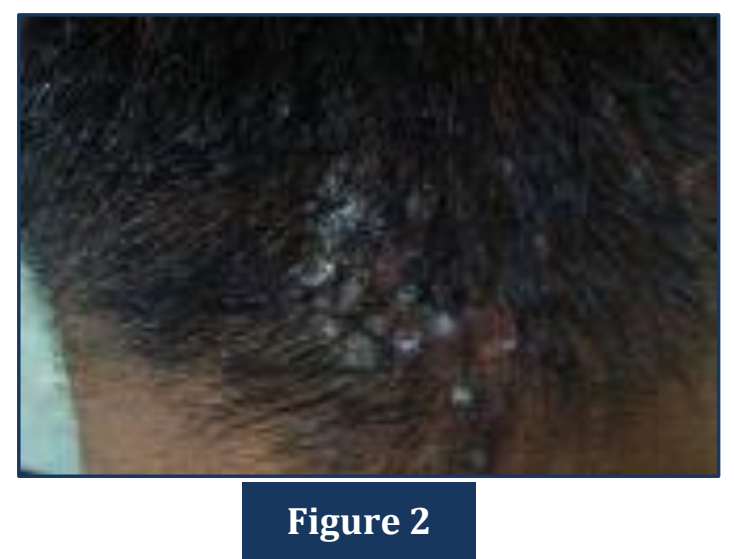

Erythematous, infiltrated plaque with dilated follicular openings with tufts containing 10 to 15 with normal hair shafts.

HISTOPATHOLOGICAL EVALUATION: The histopathological studies for both the patients showed epidermal hyperkeratosis with parakeratosis. Follicular plugging was present. Tufts of hair surrounded by perifollicular inflammation of plasma cells, lymphocytes and neutrophils around the upper portion of the hair follicles, sparing the hair root level. Some of the hair follicles were dilated 


\section{CASE REPORT}

and deformed containing abundance of debris and keratotic material. With these findings; we came to a conclusive diagnosis of Tufted Folliculitis.

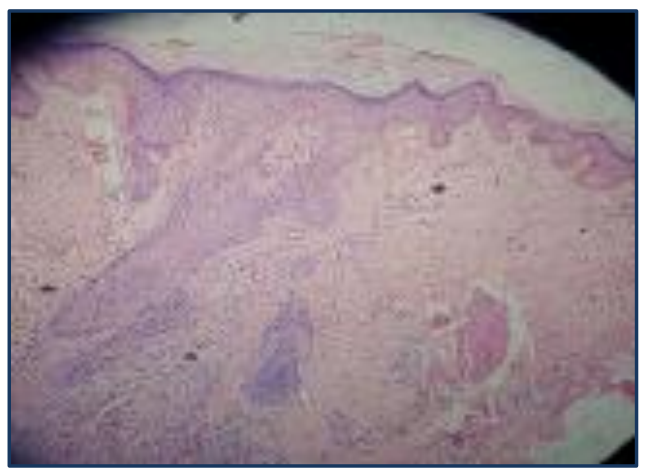

Figure 3

Histopathological section showing hyperkeratosis and parakeratosis.

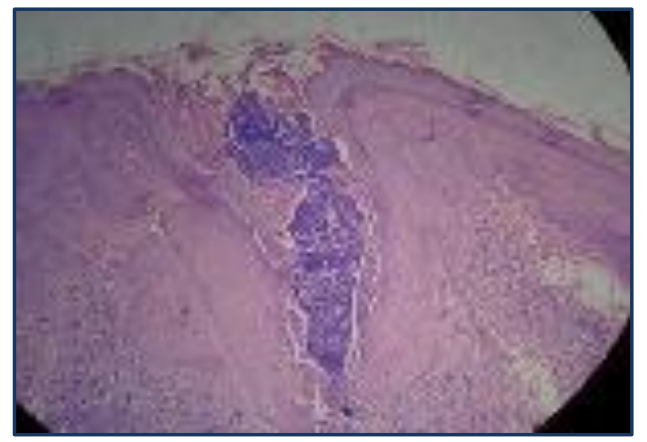

Figure 4

Dilated follicle containing keratotic material, debris, and numerous cocci. Scattered perifollicular mixed cell inflammation of plasma cells, lymphocytes, and neutrophils.

DIFFERENTIAL DIAGNOSIS: Tufted Folliculitis is an easily missed entity as it mimics various dermatological conditions that lead to cicatricial alopecia. Differential diagnosis in the early stages are: Acne keloidalis nuchae, folliculitis decalvans, follicular lichen planus and relapsing staphylococcal folliculitis.

DISCUSSION: Tufted Hair Folliculitis (THF) was described by Smith and Sanderson in $1978 .{ }^{4}$ Worldwide Tufted foliculitis is rarely encountered and documented. Most of the reported cases are from Europe and United States. Tufted Folliculitis is a progressive, recurrent, exudative and erythematous plaque with perifollicular inflammation that is characterized by multiple hair tufts arising from a single hair ostium. ${ }^{5}$ THF is more prevalent between the age groups 20 to 60 years.

It more frequently affects those in the 30s. There is a male predominance. The sites of predilection are the occipital and parietal areas. Various theories have been postulated for the pathogenesis of Tufted Folliculitis: 
1. As described by Trueb et al; staphylococcal infection is the initial causative factor. ${ }^{3}$

2. Underlying differences in follicular anatomy or host response play an important role in determining which reaction pattern occurs in an affected individual; This theory states that the development of atrophy with loss of hair structures (in folliculitis decalvans) or of hair tufts (in tufted folliculitis) depends upon the depth and destructive potential of the inflammatory process ${ }^{6}$.

3. Tufted hair folliculitis has been documented to occur following a scalp injury ${ }^{7}$, pemphigus vulgaris on the scalp 8 , and as a consequence of tinea capitis infection.

4. There is an abnormal host response to a certain presumptive superantigen ( $\mathrm{S}$. aureus, desmoglein, etc. $)^{9}$. Histopathologically; Perifollicular inflammatory around the upper portion of hair follicles with widely dilated ducts and infiltrations of plasma cells are the main features. Sometimes a ruptured follicle causes a foreign body granulomatous reaction to occur ${ }^{10}$.

Smith and Sanderson suggested that a local inflammatory process leads to destruction of superficial parts of the follicle, and ultimately causes local fibrosis and scarring1.

The management of Tufted folliculitis is very difficult. The treatment modalities include oral antibiotics and topical antiseptics. Antibiotics such as (cephalexin, cloxacillin and erythromycin diminish the inflammatory process but do not affect hair tufting. Unfortunately none have a promising outcome. Nadifloxacin, a new quinolone with broad spectrum antibacterial effect and little resistance, has been proven effective in a single patient ${ }^{11}$.

It could be used to prevent the spread of lesions or recurrence. Rifampicin is proven to be best of the antibiotics active against $\mathrm{S}$. aureus, a therapeutic modality to control the pustular phase of the disease and also to prevent possible relapses ${ }^{12}$. Definitive results have been reported after surgical excision of the involved area or with Nd YAG laser ${ }^{13,14}$. Both our patients were treated systemically with Rifampicin, Doxycycline, Paracetamol and topical Fusidic ointment and have shown good improvement after 4 weeks.

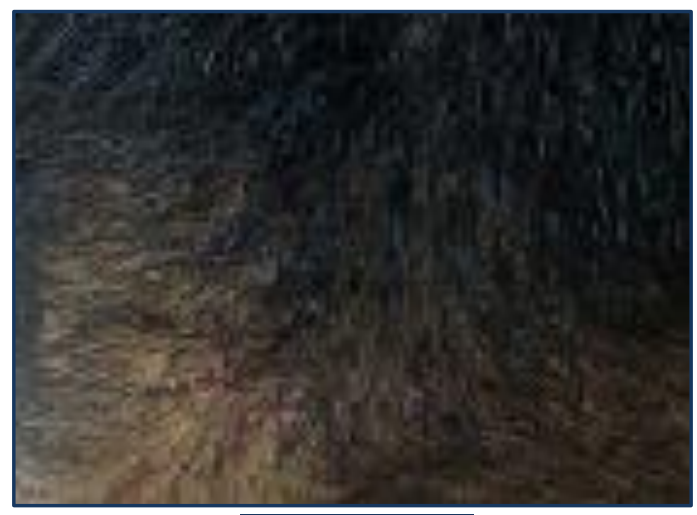

Figure 5

One week after initiating treatment with systemic Rifampicin and topical fusidic ointment. 


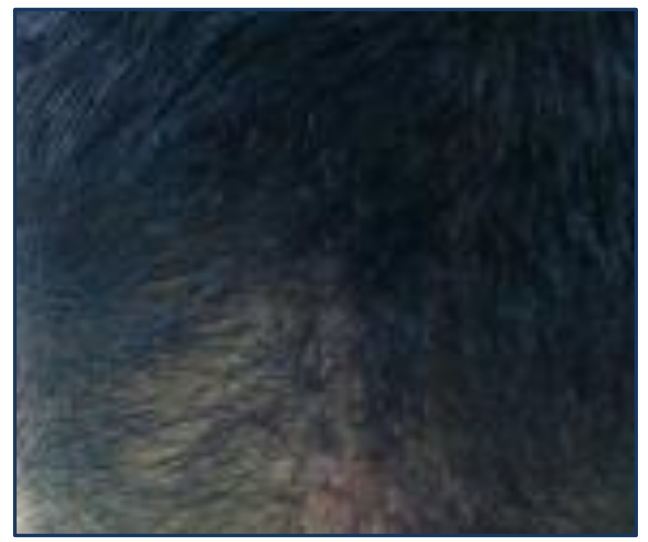

Figure 6

Two weeks after initiating treatment with Rifampicin and topical fusidic acid.

We have presented two anecdotal cases of tufted hair folliculitis; of which one was a consequence of a chronic bacterial infection that was effectively treated with Rifampicin. More clinical observations and further studies are needed to further evaluate the pathogenetic relevance of the infectious nature of this disease.

\section{REFERENCES:}

1. Annessi, G. Tufted folliculitis of the scalp: a distinctive clinicohistological variant of folliculitis decalvans. British Journal of Dermatology 138.5 (1998): 799-805.

2. Ekmekci T. R, A. Koslu. Tufted hair folliculitis causing skullcap-pattern cicatricial alopecia. Journal of the European Academy of Dermatology and Venereology 20.2 (2006): 227-229.

3. Trüeb Ralph M et al. Bündelhaar-Follikulitis. Der Hautarzt 48.4 (1997): 266-269.

4. Smith, Neil P. Tufted folliculitis of the scalp. Journal of the Royal Society of Medicine 71.8 (1978): 606.

5. Golchai J, J. Shams Gilany. TUFTED HAIR FOLLICULITIS. Medical Journal of the Islamic Republic of Iran (MJIRI) 8.2 (1994): 127-128.

6. Isomura, Iwao, Akimichi Morita, Yasuhiko Tamada. A case of tufted hair folliculitis. Journal of Dermatology 29.7 (2002): 427-430.

7. Otberg Nina et al. Folliculitis decalvans. Dermatologic Therapy 21.4 (2008): 238-244.

8. Jappe $U$ et al. Tufted hair folliculitis associated with pemphigus vulgaris. Journal of the European Academy of Dermatology and Venereology 17.2 (2003): 223-226.

9. Saijyo, Shinobu, Hachiro Tagami. Tufted hair folliculitis developing in a recalcitrant lesion of pemphigus vulgaris. Journal of the American Academy of Dermatology 38.5 (1998): $857-$ 859.

10. Powell J. J, R. P. R. Dawber, K. Gatter. Folliculitis decalvans including tufted folliculitis: clinical, histological and therapeutic findings. British Journal of Dermatology 140 (1999): 328-333.

11. Akamatsu $\mathrm{H}$ et al. Effect of nadifloxacin on neutrophil functions. The Journal of International Medical Research 23.1 (1994): 19-26.

12. Pranteda G et al. Tufted hair folliculitis: complete enduring response after treatment with rifampicin. Journal of Dermatological Treatment 15.6 (2004): 396-398. 
13. Ramos M. Luz, M. A. Munoz-Perez, A. Ports, M. Ortega, F. Camacho. Acne keloidalis nuchae and tufted hair folliculitis. Dermatology 194, no. 1 (1997): 71-73.

14. Parlette Eric C, Nathan Kroeger, E. Victor Ross. Nd: YAG laser treatment of recalcitrant folliculitis decalvans. Dermatologic surgery 30, no. 8 (2004): 1152-1154.

\section{AUTHORS:}

1. Sowmya Naga Dogiparthi

2. M. Krishnakanth

3. S. Adikrishnan

4. Adithya B.

5. Sudha R.

\section{PARTICULARS OF CONTRIBUTORS:}

1. Resident, Department of Dermatology, Sri Ramachandra Medical College and Research Institute.

2. Assistant professor, Department of Dermatology, Sri Ramachandra Medical College and Research Institute.

3. Associate Professor, Department of Dermatology, Sri Ramachandra Medical College and Research Institute.
4. Assistant Professor, Department of Dermatology, Sri Ramachandra Medical College and Research Institute.

5. Professor and HOD, Department of Dermatology, Sri Ramachandra Medical College and Research Institute.

\section{NAME ADDRESS EMAIL ID OF THE CORRESPONDING AUTHOR:}

Dr. Sowmya Naga Dogiparthi, Sai Kuteeram, New No.16,

Old No.13, $9^{\text {th }}$ Lane,

Sastrinagar, Adayar, Chennai-20.

E-mail: sowmy88688@gmail.com

Date of Submission: 01/03/2014.

Date of Peer Review: 03/03/2014.

Date of Acceptance: 10/03/2014.

Date of Publishing: 25/03/2014. 